\title{
Effect of Food Status on the Gastrointestinal Transit of Amphotericin B-Containing Solid Lipid Nanoparticles in Rats
}

\author{
Hilda Amekyeh, ${ }^{1}$ Nashiru Billa, ${ }^{1,3}$ Kah-Hay Yuen, ${ }^{2}$ and Sheau Chin Sherlyn Lim ${ }^{2}$
}

Received 29 July 2015; accepted 15 October 2015; published online 28 October 2015

\begin{abstract}
Amphotericin B (AmB) is poorly absorbed from the gastrointestinal tract. Recent studies have suggested enhanced drug absorption from solid lipid nanoparticles (SLN). Little is known of the fate of $\mathrm{AmB}$ absorption within the gastrointestinal tract, and no gastrointestinal transit study has yet been performed on AmB-containing nano-formulations. We aimed to investigate the effect of food on the gastrointestinal transit properties of an AmB-containing SLN in rats. Three SLNs containing AmB, paracetamol, or sulfasalazine were formulated using cocoa butter and beeswax as lipid matrices and simultaneously administered orally to Sprague-Dawley rats. Paracetamol and sulfapyridine were used as marker drugs for estimating gastric emptying and cecal arrival, respectively. The pharmacokinetic data generated for paracetamol and sulfapyridine were used in estimating the absorption of the AmB SLNs in the small and large intestines, respectively. A delayed rate of AmB absorption was observed in the fed state; however, the extent of absorption was not affected by food. Specifically, the percentages of AmB absorption during the fasted state in the stomach, small intestine, and colon were not significantly different from absorption within the respective regions in the fed state. In both states, however, absorption was highest in the colon and appeared to be a combination of absorption from the small intestine plus absorption proper within the colon. The study suggests that AmB SLN, irrespective of food status, is slowly but predominantly taken up by the lymph, making the small intestine the most favorable site for the delivery of the AmB SLNs.
\end{abstract}

KEY WORDS: amphotericin B; gastrointestinal transit; paracetamol; solid lipid nanoparticles; sulfapyridine.

\section{INTRODUCTION}

Amphotericin B (AmB) is a polyene antimycotic agent with broad spectrum activity and is very effective in treating life-threatening fungal infections. Despite its benefits, AmB has poor aqueous solubility and also causes nephrotoxicity, which could lead to permanent renal impairment especially if co-administered with other drugs that cause renal toxicity. Currently, AmB is principally delivered intravenously but this route of administration is associated with adverse effects like fever, chills, rigors, malaise, headache, generalized aches, nausea, vomiting, and hypoxia (1). There is evidence that lipidbased AmB formulations present reduced renal toxicities (24) and enhanced bioavailability (compared with AmB suspensions) when delivered orally (3-5). One such lipid-based delivery system is solid lipid nanoparticles (SLNs), which are submicron (10 to $1000 \mathrm{~nm}$ ) particles formulated from biocompatible solid lipids to produce a matrix within which a drug

\footnotetext{
$\overline{{ }^{1} \text { School of Pharmacy, University of Nottingham, Malaysia Campus, }}$ Semenyih, 43500, Selangor, Malaysia.

${ }^{2}$ School of Pharmaceutical Sciences, University of Science Malaysia, 11800, Minden, Penang, Malaysia.

${ }^{3}$ To whom correspondence should be addressed. (e-mail: Nashiru.Billa@nottingham.edu.my)
}

may be molecularly dispersed. Improved bioavailability, safety, and protection against degradation of the drug payload in the stomach are some of the desirable attributes associated with oral delivery of SLNs. Furthermore, it is possible to tailor formulations to ensure controlled release of the payload from the SLNs (6-10). Uptake of intact SLNs via the lymphatic route into blood has been reported in some studies after oral and duodenal administrations of SLNs to rats. In this regard, the involvement of the Peyer's patches in the uptake of SLN has been indicated $(11,12)$. Prior to their uptake, SLNs may adhere to the gut wall, which prolong their residence time within the gut. An increase in residence time has been linked to improved bioavailability of drugs. SLNs may also be emulsified by lipases to produce surface active mono- and diacylglycerols which can solubilize the incorporated drug prior to uptake and emptying in the lymph, a process which has been reported to augment absorption (13).

The intake of food can considerably alter the bioavailability of orally administered drugs. This alteration may manifest as a result of changes in the drug dissolution process prior to absorption, changes in GI residence time of the dosage form, or modification in membrane permeability of the drug. Furthermore, the type and quantity of food, as well as the time interval between food intake and administration of the dosage form, may reduce, delay, increase, or accelerate drug 
absorption. In some cases, there is no effect on drug absorption. Furthermore, different formulations or dosage forms of the same drug may be affected differently by food $(14,15)$. A study in humans showed that the small intestinal transit time (SITT) of dosage forms is more consistent than the gastric transit time (GTT) and that the former was not affected by the nature (physical state or size) of the administered dosage form or by the presence of food in the stomach. On the other hand, GTT was affected by the aforementioned factors (16). It is therefore necessary that studies involving food status on the absorption of drugs from their various dosage forms are conducted in order to allow appropriate assessment of the resulting pharmacokinetic data, which in turn can be used to optimize the formulation to maximize bioavailability.

In a previous work, we have successfully developed an AmB-containing SLN formulation, subjected the same to extensive characterization (17-19) and we have performed a pilot GI transit study of the formulation in fasted rats (5). In the present paper, we describe the pharmacokinetics of AmB as a consequence of food status and the GI transit of AmBcontaining SLNs using an indirect estimation method (20-22). Paracetamol (PAR) was used to estimate the GTT of the SLNs based on the rate of its appearance in the blood following oral administration of PAR-containing SLNs (23) similarly formulated as the AmB and sharing identical physical characteristics (5). Sulfapyridine (SP) is a metabolic product from the activity of colonic flora on sulfasalazine (SSZ) and is very quickly and almost completely absorbed from the colon into the blood and, thus, served as an estimate of the arrival time of the SSZ-containing formulation at the cecum $(24,25)$, also similarly formulated as AmB SLNs (5).

PAR and SP have been used as marker drugs to estimate the gastric emptying (GE) and orocecal transit times of pelletfilled capsules in humans $(20,26)$ and liquids in monkeys $(21,22)$, as well as in our previous pilot study (5). These marker drugs provide a cheaper, safer, and more appropriate alternative to the use of gamma scintigraphy for evaluating GI transit of pharmaceutical formulations in experimental animals like rats.

\section{MATERIALS AND METHODS}

\section{Materials}

Cocoa butter (JB Cocoa Sdn Bhd, Johor, Malaysia), beeswax (Acros Organic, New Jersey, USA), amphotericin B (Nacalai Tesque, Inc., Kyoto, Japan), and sulfasalazine (Tokyo Chemical Industry Co. Ltd, Japan) were purchased from the respective manufacturers. Paracetamol and sulfapyridine were purchased from Sigma-Aldrich (Sigma-Aldrich Co. LLC., Missouri, USA), lecithin soy and sodium cholate were obtained from MP Biomedicals (Illkirch, France). Chloroform, ethyl acetate, and methanol were purchased from Fisher Scientific (Loughborough, UK). All other reagents and chemicals used were of analytical grade or high-performance liquid chromatography (HPLC) grade.

\section{Preparation of SLNs}

The three types of SLNs (containing AmB, PAR, or SSZ) were prepared as previously described $(5,17)$. Briefly, $50 \mathrm{mg}$ of drug (AmB, PAR, or SSZ) and $120 \mathrm{mg}$ of lecithin were initially dissolved in a 40-mL mixture of chloroform and methanol at a 1:1 ratio, along with $200 \mathrm{mg}$ each of cocoa butter and beeswax. The solvent was evaporated off using Rotavapor ${ }^{\circledR}$ R-200/205 (Büchi, Switzerland) at $50^{\circ} \mathrm{C}$. The resulting druglipid matrix was melted in $20 \mathrm{~mL}$ of ethyl acetate at $70^{\circ} \mathrm{C}$ and added to $40 \mathrm{~mL}$ of $2.5 \% \mathrm{w} / \mathrm{v}$ sodium cholate solution at the same temperature. The mixture was homogenized at $10,000 \mathrm{rpm}$ using an IKA T 25® homogenizer (IKA, Germany) for $6 \mathrm{~min}$. A total of $60 \mathrm{~mL}$ of water at $70^{\circ} \mathrm{C}$ was then added slowly to the mixture with continuous stirring for $20 \mathrm{~min}$ after which the organic solvent was evaporated off using the Rotavapor at $70^{\circ} \mathrm{C}$.

\section{Animals}

Six male Sprague-Dawley rats weighing $250 \pm 20$ g obtained from the Animal Holding Unit of the University of Science, Malaysia, were used for the study. The study protocol complied with the recommendations of and was approved by the Animal Ethics Committee of the University of Science, Malaysia. The animals were maintained under a 12/12 h dark/light cycle.

The rats were randomly divided into fasted and fed groups and were allowed free access to food and water prior to commencement of the study. Water was withdrawn from both groups until $2 \mathrm{~h}$ post dose administration. The fasted group was fasted overnight and allowed access to food $8 \mathrm{~h}$ after dosing with the SLNs.

\section{Drug Administration and Blood Sampling}

All the animals were given a single-dose oral gavage containing $10 \mathrm{mg} / \mathrm{kg}$ of each SLN (AmB, PAR, and SSZ) dispersed in distilled water. Blood samples $(300 \mu \mathrm{L})$ were collected from the tail end of the rats before dosing and at $0.25,0.5,0.75,1,2,4,8,12,24$, and $30 \mathrm{~h}$ post dose administration into heparinized microcentrifuge tubes. Plasma was immediately separated from the blood samples by centrifugation and then frozen until analysis.

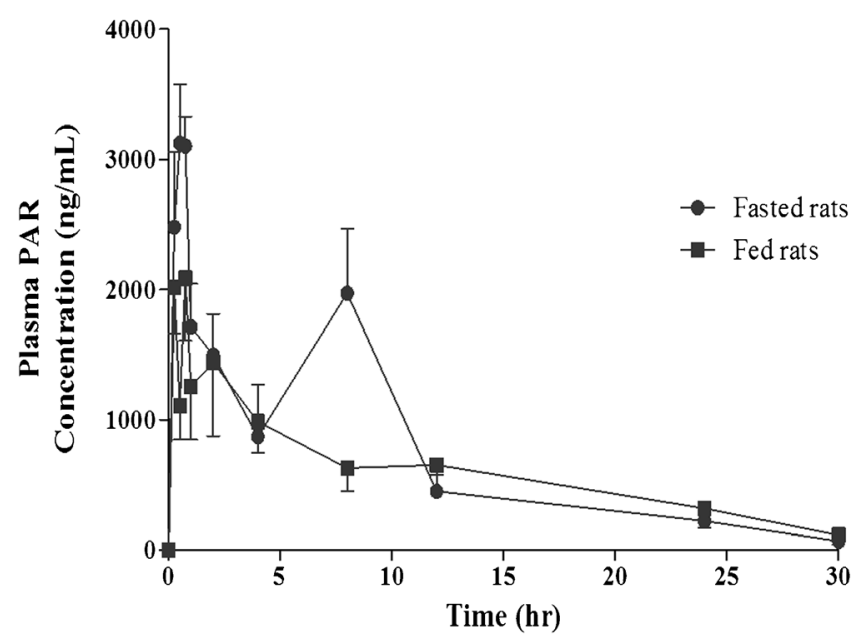

Fig. 1. Effect of food on absorption of PAR SLN in rat GI tract 
Table I. Mean Pharmacokinetic Parameters for PAR After Simultaneous Oral Gavage of SLNs Containing AmB, PAR, and SSZ Each at an Equivalent Dose of $10 \mathrm{mg} / \mathrm{kg}\left(\mathrm{mean}_{ \pm} \mathrm{SD}, n=3\right)$

\begin{tabular}{lccrr}
\hline Group & Dose $(\mathrm{mg} / \mathrm{kg})$ & $T_{\max }(\mathrm{hr})$ & $C_{\max }(\mathrm{ng} / \mathrm{mL})$ & $\mathrm{AUC} \mathrm{C}_{0-30}(\mathrm{ng} \cdot \mathrm{hr} / \mathrm{mL})$ \\
\hline Fasted & 10 & $0.5 \pm 0.25$ & $3616.3 \pm 68.4^{*}$ & $21,786 \pm 5879.8$ \\
Fed & 10 & $0.75 \pm 0.29$ & $2383.3 \pm 747.9$ & $18,135.7 \pm 5712.2$ \\
\hline
\end{tabular}

$A U C$ area under the curve

${ }^{*} p<0.05$ - the difference between fasted and fed groups is statistically significant

\section{Plasma Sample Treatment}

A $150 \mu \mathrm{L}$ aliquot of internal standard (IS) solution (piroxicam in a methanol/ethanol (1:1) mixture) was added to $100 \mu \mathrm{L}$ of rat plasma. The methanol/ethanol mixture served as a solvent for the IS and a deproteinizing agent for the plasma. The samples were then vortex-mixed for $5 \mathrm{~min}$ and centrifuged at $14,000 \mathrm{rpm}$ for $10 \mathrm{~min}$. The supernatants obtained were filtered through a $0.20-\mu \mathrm{m}$ filter and then analyzed for drug (AmB, PAR, and SP) content using a validated HPLC method (27).

\section{Estimation of Gastric Transit Time}

The parameters for estimating the time elapsed for GE or GTT were obtained from the plasma PAR absorption-time profiles and based on the assumption that the percentage of PAR absorbed was directly related to the percentage of SLNs emptied from the stomach to the duodenum (20). This served as a fairly good estimate since all three types of SLNs shared identical physical characteristics and therefore the assumption was that all three SLNs would transit similarly within the GI tract (5). The time for complete emptying of the SLNs $\left(T_{90 \mathrm{P}}\right)$ from the stomach was estimated using the time for $90 \%$ of PAR absorption in the small intestine, which was also considered to be the GTT. Another parameter obtained from the plasma PAR absorption-time profile was the time for $10 \%$ PAR absorption $\left(T_{10 \mathrm{P}}\right)$, which signaled the arrival of the SLNs at the small intestine.

\section{Estimation of Small Intestine Transit Time}

The SITT was estimated as the time difference between the cecal arrival of the SLNs and the start of emptying of the SLNs into the small intestines $\left(T_{10 \mathrm{P}}\right)$. SITT was therefore approximately the difference between

Table II. Individual Values for $T_{10 \mathrm{P}}, T_{90 \mathrm{P}}$, and GTT Estimated from Plasma PAR Profile $(n=3)$

\begin{tabular}{lccccc}
\hline \multirow{2}{*}{ Rat no. } & \multicolumn{2}{c}{ Fasted } & & \multicolumn{2}{c}{ Fed } \\
\cline { 2 - 3 } \cline { 5 - 6 } & $T_{10 \mathrm{P}}(\mathrm{h})$ & $T_{90 \mathrm{P}}(\mathrm{GTT})(\mathrm{h})$ & & $T_{10 \mathrm{P}}(\mathrm{h})$ & $T_{90 \mathrm{P}}(\mathrm{GTT})(\mathrm{h})$ \\
\hline 1 & 0.16 & 1.56 & & 0.08 & 1.74 \\
2 & 0.18 & 2.38 & & 0.16 & 2.38 \\
3 & 0.09 & 1.19 & & 0.10 & 2.63 \\
Mean & 0.14 & 1.71 & & 0.11 & 2.25 \\
SD & 0.04 & 0.61 & & 0.04 & 0.46 \\
\hline
\end{tabular}

GTT gastric transit time, $S D$ standard deviation
$T_{10 \mathrm{~S}}$ (the time taken for $10 \% \mathrm{SP}$ absorption in the cecum) and $T_{10 \mathrm{P}}$.

\section{Estimation of Cecal Arrival Time and Colonic Transit Time}

Data obtained from the plasma SP concentration-time curves were used to determine the cecal arrival time (CAT) of the AmB SLNs. The CAT by definition is the time taken for the SLNs to arrive at the cecum and has been estimated as the time for the initial detection of SP in the plasma using the indirect method of estimation (25). Due to the time lapse in SP production from SSZ released from SSZ SLNs reaching the cecum, CAT was estimated to be approximately $T_{10 \mathrm{~s}}$. $T_{10 \mathrm{~s}}$ serves as a better estimate for CAT of the SLNs than the first SP detection in plasma as the latter may be mainly due to free SSZ released from the SLN rather than released SSZ from the intact SSZ SLNs within the cecum. The colonic transit time (CTT) was estimated as the time for $90 \% \mathrm{SP}$ absorption $\left(T_{90 \mathrm{~S}}\right)$.

\section{Estimation of AmB Absorption in the Stomach, Small Intestine, and Colon}

The percentages of AmB SLNs absorbed in the stomach, small intestine, and the colon were estimated from the plasma AmB absorption-time profiles using the respective transit times (GTT and SITT). The percentage of AmB absorbed during gastric transit or GTT was estimated from the time of dose administration to $T_{10 \mathrm{P}}$. The time from $T_{10 \mathrm{P}}$ to $T_{10 \mathrm{~s}}$ or SITT was used to estimate the percentage absorption in the small intestine while the remaining percentage of AmB SLNs absorbed after this point was considered as the summation of both colonic and progressed lymphatic absorption.

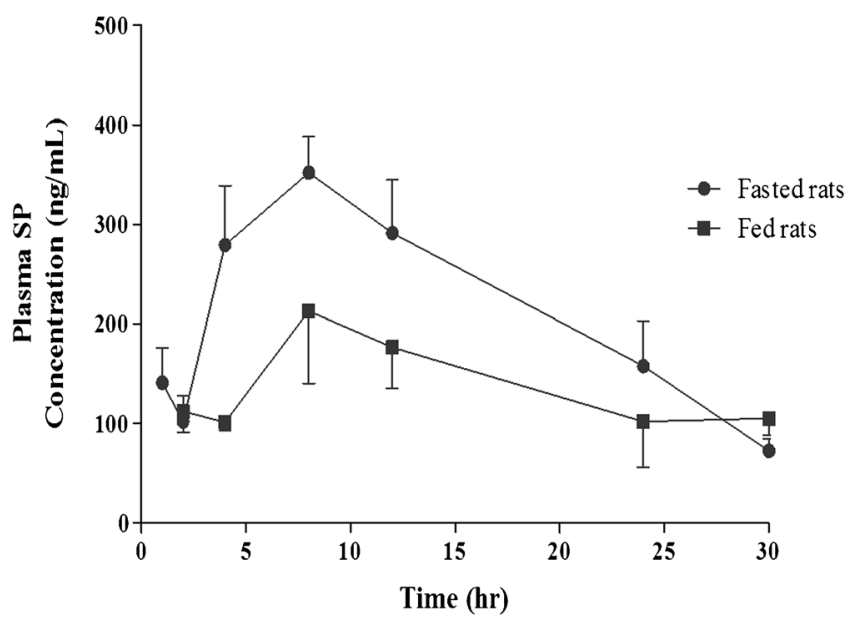

Fig. 2. Effect of food on absorption of SP (from SSZ) in rat colon 
Table III. Individual Values of $T_{\max }, T_{10 \mathrm{~S}}$, CAT, and SITT Under Fasted and Fed States Estimated from SP Plasma Profiles $(n=3)$

\begin{tabular}{|c|c|c|c|c|c|c|c|c|}
\hline \multirow[t]{2}{*}{ Rat no. } & \multicolumn{4}{|c|}{ Fasted } & \multicolumn{4}{|c|}{ Fed } \\
\hline & $T_{\max }(\mathrm{h})$ & $T_{10 \mathrm{~s}}(\mathrm{CAT})(\mathrm{h})$ & SITT (h) & CTT (h) & $T_{\max }(\mathrm{h})$ & $T_{10 \mathrm{~S}}(\mathrm{CAT})(\mathrm{h})$ & SITT (h) & CTT (h) \\
\hline 1 & 8 & 1.92 & 1.76 & 19.9 & 8 & 0.92 & 0.84 & 23.6 \\
\hline 2 & 12 & 0.93 & 0.75 & 13.9 & 12 & 1.42 & 1.26 & 22.7 \\
\hline 3 & 8 & 2.54 & 2.45 & 18.8 & 12 & 3.36 & 3.26 & 27.5 \\
\hline Mean & 9.3 & 1.80 & 1.65 & $15.5^{*}$ & 10.7 & 1.90 & 1.79 & 24.6 \\
\hline SD & 2.3 & 0.81 & 0.86 & 3.2 & 2.3 & 1.29 & 1.29 & 2.6 \\
\hline
\end{tabular}

$C A T$ cecal arrival time, SITT small intestinal transit time, $C T T$ colonic transit time, $S D$ standard deviation $* p<0.05$ - the difference between fasted and fed groups is statistically significant

\section{Statistical Analysis}

The data have been presented as mean \pm SD where indicated. Statistical analyses were performed using GraphPad Prism 5 software. A statistically significant difference was considered when the $p$ value $<0.05$, using paired $t$ test for fasted and fed groups.

\section{RESULTS AND DISCUSSION}

\section{Gastric Transit of the AmB SLNs}

The mean plasma PAR concentration-time profiles in both fasted and fed rats are presented in Fig. 1, and the pharmacokinetic data obtained from the profiles are presented in Table I. In both the fasted and fed states, we observed a rapid PAR absorption albeit slower rate in the fed state. Furthermore, multiple peaks were observed within the first $4 \mathrm{~h}$ of the PAR concentration time profile for the fed rats, possibly due to the effect of food. A later peak occurred at $8 \mathrm{~h}$ post dose administration in the fasted rats and can be attributed to the delayed appearance of PAR in the plasma due to possible uptake of intact SLNs by Peyer's patches via the lymph $(11,12)$ followed by assimilation and PAR release into the blood. This is a slow process and therefore expected to manifest late. In the fed rats, however, no such sharp peak at a later time is observed as the presence of food slowed the absorption rate of the intact SLNs. PAR has better aqueous

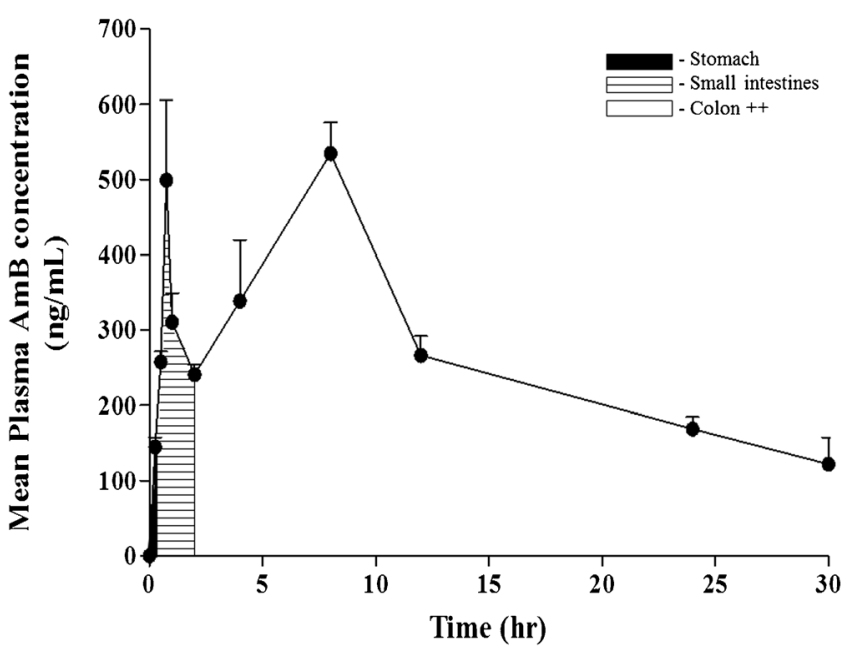

Fig. 3. Absorption of AmB SLNs in the fasted rats solubility as compared with AmB or SSZ hence PAR favorably partitions out of the hydrophobic lipid matrix of the SLNs into the more aqueous GI fluids leaving most of the absorbed SLNs in the fed rats containing very minimal amounts of the drug. The presence of food caused an insignificant reduction in $C_{\max }(p=0.05)$ and area under the curve (AUC) $)_{0-30}$ $(p=0.484)$ and a delay in $T_{\max }(p=0.725)$ of PAR absorption. It is clear from the mean values that a delayed $T_{\max }$ was caused by the presence of food through a slower gastric emptying process of the SLNs. In both fasted and fed groups, no lag time in PAR absorption was observed; however, this phenomenon is most likely a result of the rapid emptying of PAR solution released from the SLN within the administered dose and subsequent absorption in the small intestine. The absorption of this PAR solution therefore occurred almost instantly after dose administration to the rats and emptying of this solution into the small intestines occurred unobstructed. PAR absorption in humans has been found to be largely hindered in patients with pyloric stenosis and may cause a delay in GE (23); however, the data obtained in the present study suggest unimpeded emptying into the small intestine. Magnetic resonance monitoring was used in a study to estimate GE time in rats (equivalent to $T_{90 \mathrm{P}}$ ) and was found to be approximately 1.67 and $2.34 \mathrm{~h}$ after liquid (ferrofluid) and solid (ferrite powder pellets and laboratory chow) meals were orally administered, respectively (28). These values are comparable with those observed in the present study which are 1.71 and $2.25 \mathrm{~h}$ for the fasted and fed rats, respectively, as presented in Table II.

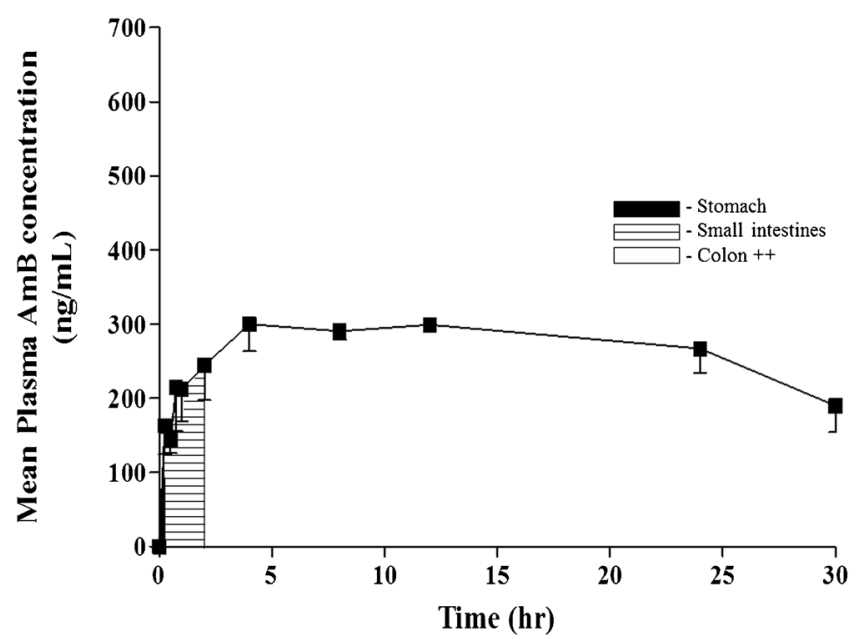

Fig. 4. Absorption of AmB SLNs in the fed rats 
Table IV. Pharmacokinetic Parameters for AmB After Simultaneous Oral Gavage of SLNs Containing AmB, PAR, and SSZ Each at an Equivalent $\mathrm{AmB}$ Dose of $10 \mathrm{mg} / \mathrm{kg}\left(\mathrm{mean}_{ \pm} \mathrm{SD}, n=3\right)$

\begin{tabular}{lllll}
\hline Group & Dose $(\mathrm{mg} / \mathrm{kg})$ & $T_{\max }(\mathrm{h})$ & $C_{\max }(\mathrm{ng} / \mathrm{mL})$ & $\mathrm{AUC} \mathrm{C}_{0-30}(\mathrm{ng} \cdot \mathrm{hr} / \mathrm{mL})$ \\
\hline Fasted & 10 & $8 \pm 0$ & $564.7 \pm 122.46^{*}$ & $7953 \pm 551.23$ \\
Fed & 10 & $9.33 \pm 4.62$ & $323.2 \pm 43.96$ & $7565.33 \pm 1390.64$ \\
\hline
\end{tabular}

$* p<0.05$ - the difference between fasted and fed groups is statistically significant

\section{Small Intestinal Transit Time of AmB SLNs}

The mean SITT of the SLNs in the fasted rats was 1.65 and $1.79 \mathrm{~h}$ in the fed group, giving a time difference of only about $0.14 \mathrm{~h}$. This shows that transit of the SLNs in the small intestines was similar in both food states. In a study conducted by Quini et al. (28), an identical mean value of approximately $1.7 \mathrm{~h}$ was obtained as SITT in both liquid meal- and solid meal-fed rats using magnetic resonance monitoring in the estimation. In humans, the same observation was made, where food did not significantly affect the SITT of orally administered dosage forms, whether a solution, pellets, or a single unit dosage form was administered $(16,20,29)$.

\section{Cecal Arrival and Colonic Transit Times of the AmB SLNs}

A number of studies on pellets or suspensions have estimated cecal arrival time as the time for the initial detection of $\mathrm{SP}$ in plasma (20-22,26). In the present study, $T_{10 \mathrm{~s}}$ was used to estimate CAT of the AmB SLNs instead of the time for the first appearance of SP in plasma which with most certainty is a result of SSZ released from the SLN as solution and arriving at the cecum ahead of the SLNs. Such an earlier arrival of the solution SSZ would lead to the hydrolysis of free SSZ and not SSZ released from intact SLNs reaching the cecum and therefore will underestimate the CAT. A more accurate CAT estimation using the first SP detection in plasma requires more frequent blood sampling, which is a constraint in the use of this indirect approach. Figure 2 and Table III show the mean SP concentration-time profiles in plasma and the individual pharmacokinetic data obtained from the profiles, respectively. Both curves showed a similar absorption pattern. Initially, there was a slight drop in plasma SP concentrations in both food statuses after which the plasma SP level rose and attained a $T_{\max }$ at $8 \mathrm{~h}$. The first concentration point in each food status is due to the absorption of SP after colonic bacterial activity on released SSZ which arrives at the colon relatively earlier than the SSZ SLNs. The slight drop in SP concentration thereafter can be attributed to a depleted source of this dissolved SSZ that arrived ahead of the SLNs due to microbial action. On the other hand, the slow rise in SP absorption in both food states was due to the time lapse required for the release of SSZ from the SSZ SLNs and microbial action by colonic bacteria when the latter was resident in the colon. The mean CAT was found to be only $0.1 \mathrm{~h}$ longer in the fed rats as compared with the fasted group $(p=0.912)$.

Methods employed in measuring CTT in humans include the use of radio-opaque markers or gamma scintigraphy and involve tracking the time it takes for ingested markers to travel along the large bowel. The mean CTT obtained in one human study with and without the use of barium paste were $74.9 \pm 49.3$ and $85.7 \pm 46.1 \mathrm{~h}$, respectively (30). In a study by Enck et al. (31), a mean CTT value of $15.5 \mathrm{~h}$ was obtained in rats. In that study, a carmine red solution was infused into the cecum of the rats and the time for the first discharge of a redcolored fecal pellet was considered as CTT. Usually in CTT determination, subjects or animals are allowed to maintain their usual dietary intake and medications if any, provided the latter does not interfere with intestinal motility. In the present study, the mean CTT in the fasted rats was found to be $15.5 \mathrm{~h}$, which was statistically shorter $(p=0.04)$ than that observed for the fed rats $(24.6 \mathrm{~h})$. The difference in the values obtained in the present study and that from the study by Enck et al. (31) is due to the methods employed by both groups. The CTT estimation by Enck et al. (31) was based on infusing of the marker solution directly into the cecum, which has the potential of staining the fecal matter already present before the study commenced.

\section{Estimated AmB Absorption in the Stomach, Small Intestine, and Colon}

All three types of particles were spherical in shape and appeared to have somewhat rough surfaces as previously reported (5). In a previous study, we have shown that AmB is molecularly dispersed with the lipid matrix of the SLNs (19)

Table V. Estimated Percentage Absorption of AmB from SLNs in the Stomach, Small Intestines, and Colon $(n=3)$

\begin{tabular}{|c|c|c|c|c|c|c|}
\hline \multirow[t]{2}{*}{ Rat no. } & \multicolumn{3}{|c|}{ Fasted } & \multicolumn{3}{|c|}{ Fed } \\
\hline & Stomach (\%) & Small intestines $(\%)$ & Colon $^{++}(\%)$ & Stomach (\%) & Small intestines (\%) & Colon $^{++}(\%)$ \\
\hline 1 & 3.4 & 53.5 & 43.1 & 1.9 & 19.8 & 78.3 \\
\hline 2 & 3.4 & 32.4 & 64.2 & 3.8 & 40.4 & 55.8 \\
\hline 3 & 1.5 & 46.4 & 52.1 & 4.0 & 51.3 & 44.7 \\
\hline Mean & 2.8 & 44.1 & 53.1 & 3.2 & 37.2 & 59.6 \\
\hline $\mathrm{SD}$ & 1.1 & 10.7 & 10.6 & 1.2 & 16.0 & 17.1 \\
\hline
\end{tabular}

$S D$ standard deviation 
and that all three SLNs shared identical physical characteristics (5) and therefore expected to respond to the hydrodynamics of the GI similarly. A molecularly dispersed arrangement of $\mathrm{AmB}$ within the lipid matrix favors prior interaction of the lipid with the intestinal epithelia and assimilation of the SLNs in Peyer's patches $(10,11,13)$. The mean plasma AmB concentration-time profiles in the fasted and fed rats are depicted in Figs. 3 and 4 respectively, and the pharmacokinetic data obtained from them are shown in Table IV. The first and second boundaries demarcated in Figs. 3 and 4 represent $T_{10 \mathrm{P}}$ and $T_{10 \mathrm{~S}}$, respectively, and the various regions within the profiles correlate the extent of absorption of $\mathrm{AmB}$ within these respective regions.

As shown in Figs. 3 and 4, the plasma concentration versus time curves obtained for the absorption of $\mathrm{AmB}$ from the AmB SLNs under fasted and fed conditions presented two peaks, with those from the fasted rats being more prominent and occurring at 0.75 and $8 \mathrm{~h}$ while in the fed group, 4 and $12 \mathrm{~h}$, respectively. The presence of food slowed down the rate of AmB absorption, similarly to that observed in the absorption of PAR from SLNs (Fig. 1). Using TEM analysis, intact SLNs have been observed circulating the lymph and blood 30 min post duodenal administration of SLNs to rats (11). We believe that the appearance of the second peak is attributed to the process of SLN uptake by Peyer's patches and then assimilation of AmB in the lymph, prior to emptying in the blood. This process is thus slow, and hence, AmB only appears in blood after a finite time. The latter is the basis for "colon" ${ }^{++}$" designated in Figs. 3 and 4. Absorption of the AmB SLN in the colon ${ }^{++}$regions is attributable to a combination of absorption processes in the colon per se as well as the continued absorption process via the lymph in the small intestines explained earlier. This means that plasma AmB concentration post CAT is not attributed solely to colonic drug absorption. At the present time, we have not established the proportion of colonic only AmB absorption against the same via the lymph. However, we believe that the incidence of the second peak culminates from prolonged absorption of AmB due to slowed transit SLNs within the gut (7).

The $C_{\max }$ for $\mathrm{AmB}$ obtained from the fasted rats is well defined and significantly higher $(p=0.03)$ than that in the fed group, signifying a more rapid rate of absorption of $\mathrm{AmB}$ in the fasted state. There was no significant difference $(p=0.854)$ between the $\mathrm{AUC}_{0-30}$ in the two groups. The absence of sharp peaks in the AmB absorption profile in the fed rats (Fig. 4) and relatively steady plasma concentration during the study period is a feature that can be exploited for controlled AmB delivery.

Table $\mathrm{V}$ shows the estimated percentage absorptions of AmB from the SLNs in the various GI regions during the fasted and fed conditions. In both cases, the highest percentage absorption occurred in the colon ${ }^{++}$region, indicating major colonic and lymphatic absorption. There was no statistically significant difference $(p>0.05)$ between the fasted and fed groups with regard to percentage absorptions in the respective GI regions (pair-wise comparisons), confirming that although the presence of food slowed the rates of absorption of AmB SLNs, the extent of absorption of AmB remained unchanged under either food status. The stomach represented the poorest percentage absorption of $\mathrm{AmB}$ albeit slightly higher in the fasted state. A significantly higher level of absorption occurred in the small intestines compared to the stomach and slightly higher in the fasted state (44.1 and $37.2 \%$ obtained for the fasted and fed rats, respectively). These findings agree with those obtained by Li et al. 2009 who showed that the ileum and colon were the main segments of absorption of quercetin-containing SLNs.

\section{CONCLUSIONS}

An indirect method was used to study the GI transit of AmB SLN, and the data obtained indicate that the presence of food slowed the rate of absorption of the AmB from the SLNs, but the extent of absorption remained essentially unchanged. Furthermore, the percentage AmB absorption was lowest in the stomach region but significantly higher in the small intestine and this process was slow to manifest and continued post cecal arrival of the SLNs. Some absorption of AmB appears to occur in the colon; however, this amount is masked by the continued lymphatic absorption process in the small intestine resulting in late $\mathrm{AmB}$ appearance in the plasma.

\section{ACKNOWLEDGMENTS}

This work was supported by the Ministry of Science, Technology and Innovation (MOSTI) (02-02-12-SF0227).

\section{REFERENCES}

1. Patel R. Amphotericin B colloidal dispersion. Expert Opin Pharmacother. 2000;1(3):475-88.

2. Risovic V, Boyd M, Choo E, Wasan KM. Effects of lipid-based oral formulations on plasma and tissue amphotericin B concentrations and renal toxicity in male rats. Antimicrob Agents Chemother. 2003;47(10):3339-42.

3. Italia JL, Yahya MM, Singh D, Ravi Kumar MNV. Biodegradable nanoparticles improve oral bioavailability of amphotericin B and show reduced nephrotoxicity compared to intravenous Fungizone. Pharm Res. 2009;26(6):1324-31.

4. Jain S, Valvi PU, Swarnakar NK, Thanki K. Gelatin coated hybrid lipid nanoparticles for oral delivery of amphotericin B. Mol Pharm. 2012;9(9):2542-53.

5. Amekyeh H, Billa N, Yuen KH, Chin SLS. A gastrointestinal transit study on amphotericin b-loaded solid lipid nanoparticles in rats. AAPS PharmSciTech. 2015;16(4):871-7.

6. Patel K, Padhye S, Nagarsenker M. Duloxetine $\mathrm{HCl}$ lipid nanoparticles: preparation, characterization, and dosage form design. AAPS PharmSciTech. 2012;13(1):125-33.

7. Luo CF, Yuan M, Chen MS, Liu SM, Zhu L, Huang BY. Pharmacokinetics, tissue distribution and relative bioavailability of puerarin solid lipid nanoparticles following oral administration. Int J Pharm. 2011;410(1-2):138-44.

8. Dwivedi P, Khatik R, Khandelwal K, Taneja I, Raju KSR, Wahajuddin, et al. Pharmacokinetics study of arteether loaded solid lipid nanoparticles: an improved oral bioavailability in rats. Int J Pharm. 2014;466 Suppl 1-2:321-27.

9. Zhang Z, Gao F, Bu H, Xiao J, Li Y. Solid lipid nanoparticles loading candesartan cilexetil enhance oral bioavailability: in vitro characteristics and absorption mechanism in rats. NanomedNanotechnol. 2012;8(5):740-7.

10. Li H, Zhao X, Ma Y, Zhai G, Li L, Lou H. Enhancement of gastrointestinal absorption of quercetin by solid lipid nanoparticles. J Control Release. 2009;133(3):238-44.

11. Bargoni A, Cavalli R, Caputo O, Fundaro A, Gasco MR, Zara GP. Solid lipid nanoparticles in lymph and plasma after duodenal administration to rats. Pharm Res. 1998;15(5):745-50. 
12. Yuan H, Chen J, Du YZ, Hu FQ, Zeng S, Zhao HL. Studies on oral absorption of stearic acid SLN by a novel fluorometric method. Colloids Surf B. 2007;58(2):157-64.

13. Müller RH, Runge S, Ravelli V, Mehnert W, Thünemann AF, Souto EB. Oral bioavailability of cyclosporine: solid lipid nanoparticles (SLN) versus drug nanocrystals. Int J Pharm. 2006;317(1):82-9.

14. Singh BN. Effects of food on clinical pharmacokinetics. Clin Pharmacokinet. 1999;37(3):213-55.

15. Welling PG. Effects of food on drug absorption. Annu Rev Nutr. 1996;16:383-415.

16. Davis SS, Hardy JG, Fara JW. Transit of pharmaceutical dosage forms through the small intestine. Gut. 1986;27(8):886-92.

17. Tan SW, Billa N, Roberts CR, Burley JC. Surfactant effects on the physical characteristics of amphotericin B-containing nanostructured lipid carriers. Colloids Surf A. 2010;372(1-3):73-9.

18. Tan SW, Billa N. Lipid effects on expulsion rate of amphotericin B from solid lipid nanoparticles. AAPS PharmSciTech. 2014;15(2):287-95.

19. Tan CSW, Billa N, Roberts CJ, Scurr DJ. Properties of an oral nanoformulation of a molecularly dispersed amphotericin B comprising a composite matrix of theobroma oil and bee's wax. Nanomaterials. 2014;4(4):905-16.

20. Peh KK, Yuen KH. Indirect gastrointestinal transit monitoring and absorption of theophylline. Int J Pharm. 1996;139(1-2):95-103.

21. Kondo H, Takahashi Y, Watanabe T, Yokohama S, Watanabe J. Gastrointestinal transit of liquids in unfed cynomolgus monkeys. Biopharm Drug Dispos. 2003;24(3):131-40.

22. Kondo H, Watanabe T, Yokohama S, Watanabe J. Effect of food on gastrointestinal transit of liquids in cynomolgus monkeys. Biopharm Drug Dispos. 2003;24(4):141-51.
23. Heading RC, Nimmo J, Prescott LF, Tothill P. The dependence of paracetamol absorption on the rate of gastric emptying. Br J Pharmacol. 1973;47(2):415-21.

24. Kellow JE, Borody TJ, Phillips SF, Haddad AC, Brown ML. Sulphapyridine appearance in plasma after salicylazosulphapyridine. Gastroenterology. 1986;91(2):396-400.

25. Staniforth DH. Comparison of orocaecal transit time assessed by the lactulose/breath hydrogen and the sulphasalazine/ sulphapyridine methods. Gut. 1989;30(7):978-82.

26. Rahman NU, Yuen KH, Woei WJ. Gastrointestinal transit monitoring and absorption of controlled-release pellets of diltiazem. Pharm Dev Technol. 2005;10(3):371-9.

27. Amekyeh H, Billa N, Yuen KH. Simultaneous HPLC assay of paracetamol and sulphapyridine as markers for estimating gastrointestinal transit of amphotericin B-containing nanoparticles in rat plasma. J Bioequivalence. 2015;1(1):104.

28. Quini CC, Américo MF, Corá LA, Calabresi MFF, Alvarez M, Oliveira RB, et al. Employment of a noninvasive magnetic method for evaluation of gastrointestinal transit in rats. J Biol Eng. 2012;6:6.

29. Yuen KH, Desmukh AA, Newton JM, Short M, Melchor R. Gastrointestinal transit and absorption of theophylline from a multiparticulate controlled release theophylline formulation. Int J Pharm. 1993;97(1-3):588-92.

30. Pomerri F, Frigo AC, Grigoletto F, Dodi G, Muzzio PC. Error count of radiopaque markers in colonic segmental transit time study. Am J Roentgenol. 2007;189(2):W56-9.

31. Enck P, Merlin V, Erckenbrecht JF, Wienbeck M. Stress effects on gastrointestinal transit in the rat. Gut. 1989;30(4):455-9. 